Persuading Consumers With Social Attitudes

Stefan Buehler and Daniel Halbheer

April 2011 Discussion Paper no. 2011-17 


$\begin{array}{ll}\text { Editor: } & \text { Martina Flockerzi } \\ & \text { University of St. Gallen } \\ & \text { School of Economics and Political Science } \\ & \text { Department of Economics } \\ & \text { Varnbüelstrasse } 19 \\ & \text { CH-9000 St. Gallen } \\ & \text { Phone } \quad+41712242325 \\ & \text { Fax } \quad+41712243135 \\ & \text { Email seps@unisg.ch } \\ & \text { School of Economics and Political Science } \\ & \text { Department of Economics } \\ & \text { University of St. Gallen } \\ & \text { Varnbüelstrasse 19 } \\ \text { Publisher: } & \text { CH-9000 St. Gallen } \\ & \text { Phone +41 71 224 23 25 } \\ & \text { Fax } \quad+41712243135 \\ & \text { http://www.seps.unisg.ch }\end{array}$




\title{
Persuading Consumers With Social Attitudes ${ }^{1}$
}

\author{
Stefan Buehler and Daniel Halbheer
}

Authors' addresses:

\author{
Stefan Buehler \\ University of St. Gallen \\ Institute of Economics (FGN-HSG) \\ Varnbüelstr. 19 \\ 9000 St. Gallen \\ Phone $\quad+41712242303$ \\ Fax $\quad+41712242670$ \\ Email_stefan.buehler@unisg.ch \\ Daniel Halbheer \\ University of Zurich \\ Department of Business Administration \\ Plattenstrasse 14 \\ 8032 Zurich \\ Phone +41446342117 \\ Fax $\quad+41712242996$ \\ Email daniel.halbheer@business.uzh.ch
}

\footnotetext{
${ }^{1}$ We thank Simon Anderson, Anette Boom, Ernst Fehr, Martin Kolmar, Gerd Mühlheusser, Ronald Peeters, Martin Peitz, Christian Thöni, and seminar participants at the University of St. Gallen, the University of Zurich, the Copenhagen University, the GEABA 2009 (Vallendar), the Workshop on Industrial Organization: Theory, Empirics and Experiments 2009 (Lecce), the SEA 2010 conference (Atlanta), the IIOC 2011 (Boston) and the IO Committee of the Verein für Socialpolitik 2011 (Vienna) for useful comments on earlier drafts. Financial support from the Swiss National Science Foundation through grants PP0012-114754 (Buehler) and PA00P1129097 (Halbheer) is gratefully acknowledged. Daniel Halbheer thanks the Department of Economics at the University of Virginia for its hospitality while some of this research was being undertaken.
} 


\begin{abstract}
This paper analyzes persuasive advertising and pricing in oligopoly if firms sell differentiated products and consumers have heterogenous social attitudes towards the consumption by others. Deriving product demand from primitives, we show that the demand-enhancing effect of persuasive advertising varies across consumers and increases in the average degree of conformity. In equilibrium, both quality and cost leaders choose higher advertising intensities and charge higher prices than their competitors. In addition, we show that an increase in the average degree of conformity among consumers reinforces asymmetries between firms.
\end{abstract}

\title{
Keywords
}

Advertising, social attitude, consumption externality, quality

\section{JEL Classification}

D11, D43, L15, L21, M37 


\section{Introduction}

Conventional theory posits that the impact of persuasive advertising on the willingness to pay does not vary across consumers. ${ }^{1}$ Johnson and Myatt (2006) refer to this type of advertising as "pure hype," noting that persuasive advertising shifts out the demand curve. However, both casual observation and empirical evidence suggest that there is a "diversity of consumer behavior toward advertising" (Gabszewicz et al., 2004, 62). ${ }^{2}$ This raises the natural question of how to reconcile the theory of persuasive advertising with the empirical evidence.

This paper proposes a model of persuasive advertising which allows the impact on the willingness to pay to vary across consumers. In line with conventional advertising theory, we start from the presumption that persuasive advertising shifts out the demand curve by increasing the perceived quality of the product. This demand shift is the direct of effect of persuasive advertising. To account for the fact that consumers often care about the choices of others (Clark and Oswald, 1998; Sobel, 2005), we further introduce consumption externalities into the analysis. Specifically, we assume that consumers have heterogenous "social attitudes" towards the consumption by others. Some consumers are "conformists" who derive a positive extra utility from the fact that other consumers buy the same product. The remaining consumers are "exclusivists" whose utility is negatively affected by others buying the same product. Since persuasive advertising shifts out the demand curve, it also affects the extra utility consumers derive from the consumption externality. This is the indirect effect of persuasive advertising which systematically varies across consumers. Adding up the direct and the indirect effect, we find that the impact of persuasive advertising on the willingness to pay generally varies across consumers.

We develop our key argument that the effect of persuasive advertising varies across consumers in two steps. We begin by considering an oligopoly model in reduced form where the intensity of persuasive advertising is chosen before

\footnotetext{
${ }^{1}$ See Bagwell (2007) for an authoritative survey of the advertising literature; von der Fehr and Stevik (1998) discuss various approaches towards modeling persuasive advertising. Rotemberg (2010) studies the role of persuasion in salesperson-customer interactions.

${ }^{2}$ For instance, for platform markets, some papers assume that consumers dislike advertising while others impose that there are both advertising avoiders and advertising lovers (see, e.g., Anderson and Coate, 2005; Gabszewicz et al., 2005; Peitz and Valletti, 2008). However, Kaiser and Song (2009) find little evidence for consumers disliking advertising.
} 
firms interact in the product market. We show that, if demand depends on a parameter capturing consumption externalities, the intensity of persuasive advertising will generally be affected by consumption externalities. However, to learn more about the impact of persuasive advertising on individual consumers' willingness to pay, it is necessary to put more structure on the model.

We consider an oligopoly model where product demand is constructed from primitives. Specifically, we assume that products are horizontally and vertically differentiated and sellers compete in persuasive advertising and pricing. Consumers have unit demand, and their product valuations are additively separable in the intrinsic consumption utility and the extrinsic utility derived from the consumption externality. To capture consumer heterogeneity, we let the taste for the preferred product variant and the social attitude vary across consumers. ${ }^{3}$ The distribution of social attitudes determines the average degree of conformity in the population.

The timing of the model is as follows. In the first stage, firms simultaneously choose their advertising intensity and thus the perceived quality of their respective products. In the second stage, firms simultaneously set prices. In the third stage, consumers form rational expectations about the demands for the products and make their purchase decisions.

We derive the following main results. First, product demand depends on the weight of the extrinsic utility (relative to the intrinsic consumption utility) and the average degree of conformity in the population. Nevertheless, product demand has the usual properties regarding prices and perceived qualities (i.e., it is decreasing in own price and increasing in own quality, and vice versa for the competitor's price and quality). Second, we find that the demand-enhancing effect of persuasive advertising varies across consumers. The result follows from the consumers' heterogenous social attitudes towards the consumption by others. Aggregating across consumers, we find that the demand-enhancing effect of persuasive advertising increases in the average degree of conformity in the population. Third, we characterize equilibrium advertising and pricing and show that both quality and cost leaders choose higher advertising intensities and charge higher prices than their competitor. This asymmetry stem from differences in the firms' quality-cost margins. Increases in the average degree of conformity, the relative weight of the extrinsic utility, and the persuasive power of advertising all reinforce the asymmetry in equilibrium.

\footnotetext{
${ }^{3}$ We also allow for "standard" consumers who are agnostic about the consumption of others and therefore derive an extrinsic utility of zero from the consumption externality.
} 
Our paper makes two contributions to the behavioral industrial organization literature. ${ }^{4}$ First, by introducing social attitudes into a persuasive advertising model, we provide a consistent explanation for the diversity of consumer behavior towards persuasive advertising. In doing so, we add to the scant literature on oligopoly models with consumption externalities, which has focused on pricing alone (Grilo et al., 2001; Amaldoss and Jain, 2005). ${ }^{5}$ Our analysis suggests that the impact of persuasive advertising on consumers is similar to informative advertising if consumers have heterogeneous social attitudes. The reason is that consumers with different social attitudes derive different extrinsic utility from consumption externalities. Second, we add to the literature on horizontal and vertical product differentiation (see, e.g., Vandenbosch and Weinberg, 1995; Anderson and de Palma, 2001; Baake and Boom, 2001). Our analysis demonstrates that heterogeneous social attitudes may reinforce the asymmetry in market shares, prices, and advertising intensities, which typically emerges in these models.

The remainder of the paper is structured as follows. Section 2 sketches the limitations of the reduced-form approach and motivates our analytical framework. Section 3 introduces our model. Section 4 derives consumer demand, studies the effects of persuasive advertising, and characterizes the equilibrium along with its comparative statics properties. Section 5 concludes.

\section{Backdrop}

To shed light on the role of social attitudes for persuasive advertising, it is natural to start from a reduced-form approach. The simplest setting is a duopoly model where one firm, say firm 1, can choose its advertising intensity $a$ in a stage proceeding product market rivalry. Suppose firm $i$ 's reduced-form demand is given by $D_{i}(\mathbf{p}, a, \mu)$, where $\mathbf{p}=\left(p_{1}, p_{2}\right)$ is the price vector and the parameter $\mu>0$ captures the importance of consumption externalities. Denoting advertising outlays by $\phi(a)$, firm 1's equilibrium profit in the advertising

\footnotetext{
${ }^{4}$ Ellison (2006) provides a survey of this literature.

${ }^{5} \mathrm{~A}$ related strand of the literature has focused on network externalities (see Farrell and Klemperer, 2007, for a survey). There are two key differences to this strand of the literature. First, network externalities stem from technical characteristics of the product, while consumption externalities relate to social attitudes of consumers. Second, network externalities are typically positive, whereas consumers may well have negative perceptions of consumption externalities.
} 
stage is given by

$$
\pi_{1}^{*}(a, \mu)=p_{1}^{*}(a, \mu) \cdot D_{1}\left(\mathbf{p}^{*}(a, \mu), a, \mu\right)-\phi(a),
$$

where the asterisk indicates optimal choices in the subsequent pricing stage.

Standard comparative statics results show that consumption externalities affect the equilibrium advertising intensity $a^{*}(\mu)=\arg \max _{a} \pi_{1}^{*}(a, \mu)$ provided that $\frac{d a^{*}}{d \mu} \neq 0$. It is thus immediate that consumption externalities generally affect profit-maximizing advertising. However, to learn more about the impact of persuasive advertising on individual consumers' willingness to pay, it is necessary to consider an oligopoly model where product demand can be constructed from primitives.

\section{Model}

We consider a model where firms choose advertising intensities and set prices for products that are horizontally and vertically differentiated. The key feature of this model is that consumers have heterogenous social attitudes regarding the consumption choices of others.

\subsection{Firms}

There are two single-product firms. Both firms $i=1,2$ choose their advertising intensity $a_{i}$ and sell their product at price $p_{i}$. Horizontal differentiation is à la Hotelling, and we assume that the firms are located at the extremes of the product spectrum at $x_{1}=0$ and $x_{2}=1$, respectively. Vertical differentiation captures the notion that advertising affects the perceived product quality. Specifically, for each product $i$, perceived product quality

$$
\theta_{i}=q_{i}+\omega a_{i}
$$

is an index weighting intrinsic quality $q_{i}$ and persuasive advertising $a_{i}$. The weighting factor $\omega>0$ reflects the persuasive power of advertising.

The advertising intensity is endogenously determined. For convenience, we suppose that advertising costs are quadratic and given by $k\left(a_{i}\right)=\beta a_{i}^{2}$, where $\beta>0$ is an exogenous parameter. The marginal cost of output with intrinsic quality $q_{i}$ is $c_{i} \geq 0$. 


\subsection{Consumers}

Consumers have unit demand. Their valuation $V_{i}$ of product $i$ reflects both intrinsic utility $v_{i}$ and extrinsic utility $e_{i} \cdot{ }^{6}$ In line with Bernheim (1994) and Clark and Oswald (1998), we assume that $V_{i}$ is additively separable in its utility components, that is,

$$
V_{i}=v_{i}+\mu e_{i},
$$

where the parameter $\mu>0$ indicates the importance of extrinsic relative to intrinsic utility.

Intrinsic Utility. A consumer buying product $i$ derives intrinsic utility

$$
v_{i}=\theta_{i}-\tau\left|x-x_{i}\right|+m-p_{i}
$$

where $\theta_{i}$ is the perceived quality, $x \in[0,1]$ is the consumer's most preferred product variant, and $m$ is income. The parameter $\tau$ measures the consumer's sensitivity to horizontal mismatch $\left|x-x_{i}\right| .^{7}$

Extrinsic Utility. A consumer's extrinsic utility depends on the number of consumers buying the same product. In line with Karni and Schmeidler (1990), we capture this number by the firms' expected equilibrium market shares and define extrinsic utility as

$$
e_{i}=\sigma\left|y-x_{i}\right|,
$$

where $y \in(0,1)$ denotes the expected demand (market share) of firm 1 and the parameter $\sigma$ captures a consumer's social attitude.

There is a continuum of social attitudes $\sigma \in[\underline{\sigma}, \bar{\sigma}]$, with $\underline{\sigma}<0<\bar{\sigma}$. Following Grilo et al. (2001) and Amaldoss and Jain (2005), consumers with $\sigma>0$ are conformists who like other consumers buying the same product, while consumers with $\sigma<0$ are exclusivists who dislike others buying the same product. Notice that the absolute value of $\sigma$ reflects the intensity of a consumer's social attitude.

Consumer Characteristics. Individual consumers are characterized by their most preferred product variant $x$ and their social attitude $\sigma$. These characteristics are drawn independently from uniform distributions over the intervals

\footnotetext{
${ }^{6} V_{i}$ reflects the consumer's complete experience of the product and therefore has the interpretation of brand value in the marketing literature (Keller and Lehmann, 2006).

${ }^{7}$ In spatial models, $\tau$ has the interpretation of transportation costs.
} 
$[0,1]$ and $[\underline{\sigma}, \bar{\sigma}]$, respectively. Independence implies that characteristics are distributed according to the bivariate uniform distribution

$$
f_{(X, \Sigma)}(x, \sigma)= \begin{cases}(\bar{\sigma}-\underline{\sigma})^{-1} & \text { if }(x, \sigma) \in T \\ 0 & \text { otherwise }\end{cases}
$$

on the rectangular space $T \equiv[0,1] \times[\underline{\sigma}, \bar{\sigma}]$. The marginal distribution of social attitudes has expectation $\mathbb{E}[\sigma]=(\underline{\sigma}+\bar{\sigma}) / 2$, which is labeled the "average degree of conformity." Individual consumer characteristics are private knowledge, and firms only know the distribution $f_{(X, \Sigma)}(x, \sigma)$.

Below, we will refer to $\tau-\mu \mathbb{E}[\sigma]$ as the sensitivity to horizontal mismatch. This expression generalizes the standard notion of the sensitivity to horizontal mismatch to our setting with social attitudes. Following Laffont et al. (1998), we view $(\tau-\mu \mathbb{E}[\sigma])^{-1}$ as an index of substitutability between the two products. It is natural to assume that this index is positive, which requires $\mathbb{E}[\sigma]<\tau / \mu$.

\subsection{Timing}

In the first stage, firms simultaneously choose their advertising intensity $a_{i}$ and hence their perceived product quality $\theta_{i}$. In the second stage, firms simultaneously set prices $p_{i}$. In the third stage, consumers form rational expectations about demand $y$ and make their purchase decisions.

\section{Analysis}

In this section, we derive the demand functions, study the interaction of social attitudes and persuasive advertising, and derive the product market equilibrium.

\subsection{Demand}

We now derive product demands $D_{i}(\mathbf{p}, \boldsymbol{\theta})$ as a function of prices $\mathbf{p}=\left(p_{1}, p_{2}\right)$ and perceived qualities $\boldsymbol{\theta}=\left(\theta_{1}, \theta_{2}\right)$. For convenience, we assume that the market is covered and impose that consumers have rational expectations about $y$ (Katz and Shapiro, 1985).

To construct the set of types $B(y)$ who buy product 1 conditional on the belief $y$, we solve the indifference condition $V_{1}=V_{2}$ to obtain the cut-off value

$$
x(\sigma \mid y)=\frac{\tau+\left(\theta_{1}-p_{1}\right)-\left(\theta_{2}-p_{2}\right)+\mu \sigma(2 y-1)}{2 \tau} .
$$




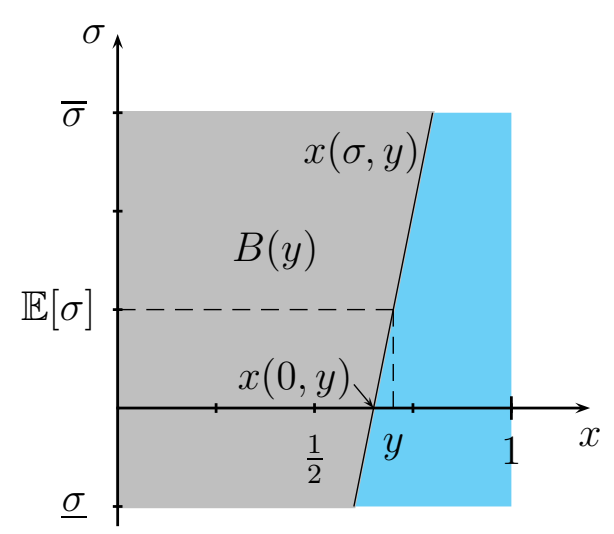

(A)

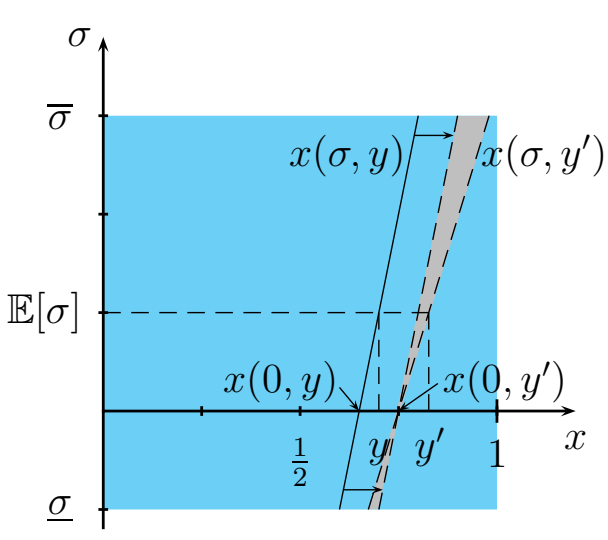

(B)

Figure 1: Demands for $y>\frac{1}{2}$ (in Panel A, the shaded area indicates types that buy from firm 1) and individual demand changes when the perceived quality of product 1 changes (in Panel B).

For given $y$, the cut-off value $x(\sigma \mid y)$ traces out the points of indifference in the type space $T$ when going from the most exclusivist attitude $\underline{\sigma}$ to the most conformist attitude $\bar{\sigma}$. The slope of this "indifference curve" is determined by the belief $y$ and given by

$$
\frac{d \sigma(x \mid y)}{d x}=\frac{2 \tau}{\mu(2 y-1)}
$$

which is positive for $y>1 / 2$ and negative for $y<1 / 2$. For $y>1 / 2$, the indifference curve is upwards-sloping because conformists can compensate the disutility from horizontal mismatch with extra utility from the consumption externality. Using the cut-off value in (4), the set of types who buy product 1 is characterized by

$$
B(y) \equiv\left\{(x, \sigma) \mid x-\frac{\mu \sigma(2 y-1)}{2 \tau} \leq \frac{\tau+\left(\theta_{1}-p_{1}\right)-\left(\theta_{2}-p_{2}\right)}{2 \tau}\right\} .
$$

This set is illustrated in Panel A of Figure 1.

Since consumers form rational expectations, the belief $y$ regarding firm 1's equilibrium demand must satisfy the fixed point condition

$$
y=\frac{1}{(\bar{\sigma}-\underline{\sigma})} \int_{\underline{\sigma}}^{\bar{\sigma}} \int_{0}^{1} \mathbf{1}_{B(y)} d x d \sigma,
$$

that is, the expected demand $y$ for product 1 must be equal to the actual demand (given $y$ ). 
Proposition 1 (Demand). Suppose $x(\sigma \mid y) \in(0,1)$ for all social attitudes $\sigma$ and beliefs $y \in(0,1)$. Then, for given prices $\mathbf{p}$ and perceived qualities $\boldsymbol{\theta}$, firm i's demand is

$$
D_{i}(\mathbf{p}, \boldsymbol{\theta})=\frac{1}{2}+\frac{\left(\theta_{i}-\theta_{j}\right)-\left(p_{i}-p_{j}\right)}{2(\tau-\mu \mathbb{E}[\sigma])} .
$$

Proof. See Appendix.

Three remarks on Proposition 1 are in order. First, the condition on $x(\sigma \mid y)$ requires that there is an indifferent consumer for every social attitude. Intuitively, this allows us to focus on a setting where the horizontal mismatch dominates the utility from the consumption externality even for some consumers with the most extreme social attitudes.

Second, the demand $D_{i}(\mathbf{p}, \boldsymbol{\theta})$ is reminiscent of demand functions in standard Hotelling models in two ways: It has the usual comparative statics properties regarding prices and qualities (i.e., it is increasing in own quality and decreasing in own price, and vice versa for the competitor's quality and price). Further, asymmetries in market shares stem only from differences in qualities or prices. Notice, in particular, that $D_{i}(\mathbf{p}, \boldsymbol{\theta})>D_{j}(\mathbf{p}, \boldsymbol{\theta})$ if and only if $\theta_{i}-p_{i}>\theta_{j}-p_{j}$. Using terminology introduced by Anderson and de Palma (2001), firm $i$ has higher demand than firm $j$ if and only if it has a higher "quality-price margin."

Third, Proposition 1 shows that social attitudes affect demand through their impact on the sensitivity to horizontal mismatch $\tau-\mu \mathbb{E}[\sigma]$. Moreover, it shows that the size of this impact depends on the importance of consumption externalities and the average degree of conformity only.

\subsection{Effects of Advertising}

Standard advertising models ignore the impact of social attitudes on demand. In our model, social attitudes have an important impact on demand and therefore on the demand-enhancing effect of advertising at both the individual and the aggregate level.

The individual effects of persuasive advertising are best illustrated using Figure 1 (Panel B). Consider an increase in firm $i^{\prime}$ s advertising intensity from $a_{i}$ to $a_{i}^{\prime}>a_{i}$. This leads to higher perceived quality $\theta_{i}$, which induces a shift of the "indifference curve" to the right. Since quality has a demand-enhancing effect, the belief $y$ is updated accordingly (i.e., $y$ increases to, say, $y^{\prime}$ ), which leads to a clockwise rotation of the indifference curve around the point $x\left(0, y^{\prime}\right)$. This 
rotation is generated by heterogeneous social attitudes: While all consumers equally benefit from the increase in intrinsic utility, the extrinsic utility of conformists and exclusivists is affected differently. The demand-enhancing effect of a quality increase is reinforced for conformists (who benefit from an increase in the demand for product 1) and weakened for exclusivists (who suffer from an increase in the demand for product 1). Therefore, conformists are more attracted to the heavily advertised product than standard consumers (with $\sigma=0$ ), whereas exclusivists are less attracted than standard consumers.

The next result highlights the diversity of consumer behavior towards persuasive advertising.

Proposition 2 (Individual Effect). Suppose that $a_{i}$ increases to $a_{i}^{\prime}>a_{i}$ and let $y^{\prime}>$ $y$ denote the corresponding beliefs. Then, the demand-enhancing effect for consumers with social attitude $\sigma$ is given by

$$
x\left(\sigma \mid y^{\prime}, a_{i}^{\prime}\right)-x\left(\sigma \mid y, a_{i}\right)=\left(\frac{\omega}{2 \tau}+\frac{\omega \mu \sigma}{2 \tau(\tau-\mu \mathbb{E}[\sigma])}\right) \int_{a_{i}}^{a_{i}^{\prime}} d \tilde{a}_{i} .
$$

Proof. See Appendix.

This result shows that persuasive advertising has a direct and an indirect effect on individual consumers. The direct effect does not depend on $\sigma$ and is thus constant across consumers (which is reflected in a parallel shift of the indifference curve). The indirect effect $\frac{\omega \mu \sigma}{2 \tau(\tau-\mu \mathbb{E}[\sigma])} \int_{a_{i}}^{a_{i}^{\prime}} d \tilde{a}_{i}$ in contrast varies across consumers. It reinforces the direct effect for conformists and may dampen or dominate the direct effect for exclusivists (which is reflected in a rotation of the indifference curve). The proposition further shows that the average degree of conformity $\mathbb{E}[\sigma]$ influences only the indirect effect of advertising.

Proposition 2 is related to Johnson and Myatt (2006). Their key idea is that individual consumers may be affected differently by informative advertising: Some are discouraged from purchasing, while others are encouraged. In our setting, the impact of advertising also varies across consumers despite its persuasive nature. Our result follows from the existence of consumption externalities rather than information transmission through advertising.

Our next result sums up the individual demand-enhancing effects across consumers to obtain the aggregate demand-enhancing effect of persuasive advertising. 
Proposition 3 (Aggregate Effect). Consider a marginal increase in firm i's advertising intensity $a_{i}$. Then, the aggregate demand-enhancing effect of persuasive advertising $\frac{\partial D_{i}(\mathbf{p}, \boldsymbol{\theta})}{\partial a_{i}}$ results from adding up the individual effects across consumers. It is increasing in the average degree of conformity, that is, $\frac{\partial^{2} D_{i}(\mathbf{p}, \boldsymbol{\theta})}{\partial \mathbb{E}[\sigma] \partial a_{i}}>0$.

Proof. See Appendix.

Proposition 3 shows that an increase in the average degree of conformity makes persuasive advertising more effective. The exception is the case where the distribution of social attitudes is symmetric around zero $(\mathbb{E}[\sigma]=0)$. In this case the sensitivity to horizontal mismatch is determined solely by $\tau$, as in the standard Hotelling model.

\subsection{Equilibrium}

In this section, we derive the subgame-perfect Nash equilibrium (SPNE) of the two-stage game and characterize its comparative statics properties.

\section{Pricing and Advertising}

Given any first-stage qualities $\boldsymbol{\theta}$, firm $i$ maximizes its product market profit and chooses its price $p_{i}$ so as to maximize $\pi_{i}(\mathbf{p})=\left(p_{i}-c_{i}\right) D_{i}(\mathbf{p}, \boldsymbol{\theta})$, which yields

$$
p_{i}(\boldsymbol{\theta})=\frac{3(\tau-\mu \mathbb{E}[\sigma])+\left(\theta_{i}-\theta_{j}\right)+2 c_{i}+c_{j}}{3} .
$$

Prices have the natural property that they are increasing in the consumers' sensitivity to horizontal mismatch, because products are perceived as being less substitutable. All else equal, equilibrium prices are decreasing in the average degree of conformity $\mathbb{E}[\sigma]$, as emphasized by Grilo et al. (2001) and Amaldoss and Jain (2005).

Firm $i^{\prime}$ s product market profit $\pi_{i}(\boldsymbol{\theta})=\left(p_{i}(\boldsymbol{\theta})-c_{i}\right) D_{i}(\boldsymbol{\theta})$ can be expressed as

$$
\pi_{i}(\boldsymbol{\theta})=\frac{\left(3(\tau-\mu \mathbb{E}[\sigma])+\left(\theta_{i}-\theta_{j}\right)-\left(c_{i}-c_{j}\right)\right)^{2}}{18(\tau-\mu \mathbb{E}[\sigma])} .
$$

Substituting the perceived qualities $\boldsymbol{\theta}$ from (1) into $\pi_{i}(\boldsymbol{\theta})$ and accounting for the cost of advertising, firm i's problem is to $\max _{a_{i}} \pi_{i}(\mathbf{a})-\beta a_{i}^{2}$, where $\mathbf{a}=\left(a_{1}, a_{2}\right)$ is the vector of advertising intensities.

The next proposition characterizes the unique subgame-perfect Nash equilibrium (SPNE) in which both firms engage in advertising. ${ }^{8}$

\footnotetext{
${ }^{8}$ The Appendix gives the conditions that ensure existence and uniqueness.
} 
Proposition 4 (Equilibrium). In the subgame-perfect Nash equilibrium where both firms engage in advertising, firm i's profit-maximizing advertising intensity and price are given by

$$
a_{i}^{*}=\frac{\omega}{2}\left(\frac{1}{3 \beta}+\frac{\left(q_{i}-c_{i}\right)-\left(q_{j}-c_{j}\right)}{9 \beta(\tau-\mu \mathbb{E}[\sigma])-\omega^{2}}\right)
$$

and

$$
p_{i}^{*}=(\tau-\mu \mathbb{E}[\sigma])+c_{i}+\frac{3 \beta(\tau-\mu \mathbb{E}[\sigma])\left(\left(q_{i}-c_{i}\right)-\left(q_{j}-c_{j}\right)\right)}{9 \beta(\tau-\mu \mathbb{E}[\sigma])-\omega^{2}} .
$$

Proof. See Appendix.

The result indicates that the aggregate advertising intensity is fixed and given by $a_{1}^{*}+a_{2}^{*}=\omega / 3 \beta$. It is fixed because the market size is normalized to unity, and it depends on the persuasive power of advertising $\omega$ and the cost parameter $\beta$ only. Thus, advertising necessarily has a business-stealing effect.

Proposition 4 contains two benchmark equilibria as special cases. The first benchmark is the case where the persuasive power of advertising tends to zero. In this case, advertising has no demand-enhancing effect, so that the firms choose not to advertise. Nevertheless, prices are affected by social attitudes, as in Grilo et al. (2001) and Amaldoss and Jain (2005). The second benchmark is the case where firms are symmetric. In this case, social attitudes affect prices only.

Proposition 4 allows us to make a number of observations (the proofs are relegated to the Appendix). To this end, we introduce the notion of firm $i^{\prime}$ s "quality-cost margin" $q_{i}-c_{i}$ (Anderson and de Palma, 2001).

Observation 1 (Asymmetric Advertising and Pricing). Suppose firm $i$ has a higher quality-cost margin than firm $j$. Then, firm $i$ has a higher advertising intensity and charges a higher price than firm $j$.

Observation 1 highlights that asymmetries in equilibrium advertising intensities and prices are driven by asymmetries in the quality-cost margins of individual firms. The result covers both quality leadership (with equal marginal costs) and cost-leadership (with equal intrinsic product qualities) as special cases. Note that both quality leaders and cost leaders invest more in advertising and sell at higher prices than their competitors.

Intuitively, Observation 1 follows from the existence of demand-markup complementarities (Athey and Schmutzler, 2001) in the product market, as the next result illustrates. 
Observation 2 (Asymmetric Profits). Suppose firm $i$ has a higher quality-cost margin than firm $j$. Then, firm $i$ has a higher markup and a higher demand than firm $j$, and hence earns a higher product market profit. Firm $i$ also earns a higher equilibrium net profit.

This observation shows that a firm with a higher quality-cost margin has a stronger incentive to invest in advertising, because the effect is more valuable thanks to a higher markup. In addition, it has a stronger incentive to increase the price, since a larger markup is more valuable thanks to larger demand. Given that equilibrium demand and markup are both larger for a firm with a higher quality-cost margin, equilibrium profits are also higher. ${ }^{9}$

The next observation characterizes the relationship between the firm's equilibrium advertising intensity and price.

Observation 3 (Price-Advertising Relation). Firm i's price and advertising intensity are positively related.

The price-advertising relationship is at the core of the advertising literature. The persuasive view of advertising holds that heavily advertised products are more expensive than less advertised products due to the consumers' higher willingness to pay (Bagwell, 2007). Observation 3 suggests that the positive relationship between the equilibrium price and advertising intensity is robust to the introduction of social attitudes.

\section{Comparative Statics}

We now provide some interesting comparative statics results of our analysis. Our focus is on marginal changes in the average degree of conformity and the persuasive power of advertising. We start with changes in the average degree of conformity.

Observation 4 (Social Attitudes). Suppose firm $i$ has a higher quality-cost margin than firm $j$. Then, a marginal increase in the average degree of conformity reinforces asymmetries in equilibrium advertising intensities and prices.

This result shows that the distribution of market shares is more asymmetric when firms face a conformist population $(\mathbb{E}[\sigma]>0)$ rather than an exclusivist population $(\mathbb{E}[\sigma] \leq 0)$.

\footnotetext{
${ }^{9}$ This result is related to earlier work by Anderson and de Palma (2001). The key difference to this paper is that our analysis allows for social attitudes among consumers.
} 
Before proceeding, we note that the comparative statics effects of changes in $\mu$, the parameter capturing the relative importance of extrinsic utility, are qualitatively the same as those of changes in $\mathbb{E}[\sigma]$. Intuitively, the result follows from the fact that both variables affect demand, and hence the equilibrium quantities, only through their impact on the sensitivity to horizontal mismatch.

Next, we analyze how the persuasive power of advertising affects equilibrium pricing and advertising intensities.

Observation 5 (Persuasive Power). Suppose firm $i$ has a higher quality-cost margin than firm $j$. Then, a marginal increase in the persuasive power of advertising reinforces asymmetries in equilibrium advertising intensities and prices.

This finding shows that the equilibrium advertising intensities of asymmetric firms are affected differently. Specifically, the firm with the higher qualitycost margin (and higher initial advertising) increases advertising, whereas the smaller firm with the lower quality-cost margin may increase or decrease ${ }^{10}$ advertising. In any case, the larger firm increases advertising more than the smaller firm, so that equilibrium advertising becomes more asymmetric.

As to prices, we find that an increase in the persuasive power of advertising reinforces the asymmetry in equilibrium behavior: The larger firm with the higher quality-cost margin (and hence the higher price) increases the price, whereas the smaller firm (with the lower price) reduces the price.

\section{Conclusion}

This paper has examined persuasive advertising and pricing in an oligopoly model with horizontal and vertical product differentiation. The key feature of this model is that a consumer's product valuation depends both on persuasive advertising and her (unobservable) individual social attitude towards the consumption of the product by others.

We have derived the following key results. First, the demand for each product depends on the weight of the extrinsic utility (relative to the intrinsic consumption utility) and the average degree of conformity in the population. Second, the demand-enhancing effect of persuasive advertising varies across consumers. The result follows from the consumers' heterogenous social attitudes towards the consumption by others. Aggregating across consumers, we have

\footnotetext{
${ }^{10}$ Note that a decrease in advertising by the smaller firm requires a very strong asymmetry in quality-cost margins, in which case the small firm can be viewed as being "marginalized."
} 
shown that the demand-enhancing effect of persuasive advertising increases in the average degree of conformity in the population. Third, both quality and cost leaders choose higher advertising intensities and charge higher prices than their competitor in equilibrium. Increases in the average degree of conformity, the relative weight of the extrinsic utility, and the persuasive power of advertising all reinforce the asymmetry in equilibrium.

Our analysis contributes to a scant behavioral industrial organization literature and suggests several avenues for future research. First, it would be interesting to examine settings where sellers may strategically manipulate the social attitudes of consumers (which are exogenous in our setting). Second, it would be natural to extend the analysis to other distributions of consumer characteristics (and higher-dimensional tastes). Third, it would be desirable to allow for the possibility of price discrimination across consumers. We hope to address these issues in future research.

\section{Appendix}

PROOF OF PROPOSITION 1: Fix $y$ and define $\underline{x}=x(\underline{\sigma}, y)$ and $\bar{x}=x(\bar{\sigma}, y)$, respectively, and recall that each point in $B \subset T$ has density $(\bar{\sigma}-\underline{\sigma})^{-1}$. Under the assumption that $x(\sigma \mid y) \in(0,1)$ for all social attitudes $\sigma$ and beliefs $y \in(0,1)$, actual demand can be expressed as $(\underline{x}+\bar{x}) / 2$. Letting $\xi \equiv \tau+\left(\theta_{1}-\theta_{2}\right)-\left(p_{1}-p_{2}\right)$ and using (4) we obtain

$$
\underline{x}=\frac{\xi+\mu \underline{\sigma}(2 y-1)}{2 \tau} \quad \text { and } \quad \bar{x}=\frac{\xi+\mu \bar{\sigma}(2 y-1)}{2 \tau},
$$

respectively. As $\mathbb{E}[\sigma]=(\underline{\sigma}+\bar{\sigma}) / 2$, we can thus express actual demand as

$$
\frac{\xi+\mu \mathbb{E}[\sigma](2 y-1)}{2 \tau},
$$

which has to be equal to expected demand $y$ under the rational expectations assumption. Solving the fixed-point condition in (5) yields

$$
y(\mathbf{p}, \boldsymbol{\theta})=\frac{\tau-\mu \mathbb{E}[\sigma]+\left(\theta_{1}-p_{1}\right)-\left(\theta_{2}-p_{2}\right)}{2(\tau-\mu \mathbb{E}[\sigma])} .
$$

By construction, $D_{1}(\mathbf{p}, \boldsymbol{\theta})=y(\mathbf{p}, \boldsymbol{\theta})$ and $D_{2}(\mathbf{p}, \boldsymbol{\theta})=1-y(\mathbf{p}, \boldsymbol{\theta})$, which establishes the result. 
PROOF OF PROPOSITION 2: Write the change in demand for consumers with social attitude $\sigma$ as

$$
\Delta x\left(\sigma \mid y, a_{i}, y^{\prime}, a_{i}^{\prime}\right) \equiv x\left(\sigma \mid y, a_{i}^{\prime}\right)-x\left(\sigma \mid y, a_{i}\right)+x\left(\sigma \mid y^{\prime}, a_{i}^{\prime}\right)-x\left(\sigma \mid y, a_{i}^{\prime}\right) .
$$

Applying the fundamental theorem of calculus, this expression can be rewritten as

$$
\Delta x(\sigma \mid \cdot)=\int_{a_{i}}^{a_{i}^{\prime}} \frac{\partial x\left(\sigma \mid y, a_{i}\right)}{\partial a_{i}} d \tilde{a}_{i}+\int_{y}^{y^{\prime}} \frac{\partial x\left(\sigma \mid y, a_{i}^{\prime}\right)}{\partial y} d \tilde{y} .
$$

Notice that (A.1) defines $y$ as a continuously differentiable function of $a_{i}$, and denote this function by $y \equiv \phi\left(a_{i}\right)$. Using integration by substitution, the second integral on the RHS of (A.2) can be written as

$$
\begin{aligned}
\int_{y}^{y^{\prime}} \frac{\partial x\left(\sigma \mid y, a_{i}^{\prime}\right)}{\partial y} d \tilde{y} & =\int_{a_{i}}^{a_{i}^{\prime}} \frac{\partial x\left(\sigma \mid \phi\left(a_{i}\right), a_{i}\right)}{\partial y} \phi^{\prime}\left(a_{i}\right) d \tilde{a}_{i} \\
& =\frac{\omega \mu \sigma}{2 \tau(\tau-\mu \mathbb{E}[\sigma])} \int_{a_{i}}^{a_{i}^{\prime}} d \tilde{a}_{i},
\end{aligned}
$$

where the last equality makes use of (4). Further substituting from (4) the expression for the first integrand on the RHS of (A.2), we have

$$
\Delta x(\sigma \mid \cdot)=\left(\frac{\omega}{2 \tau}+\frac{\omega \mu \sigma}{2 \tau(\tau-\mu \mathbb{E}[\sigma])}\right) \int_{a_{i}}^{a_{i}^{\prime}} d \tilde{a}_{i},
$$

which establishes the result.

PROOF OF PROPOSITION 3: Aggregating demand changes across consumers yields

$$
\frac{1}{\bar{\sigma}-\underline{\sigma}} \int_{\underline{\sigma}}^{\bar{\sigma}} \Delta x(\sigma \mid \cdot) d \sigma=\frac{\omega}{2(\tau-\mu \mathbb{E}[\sigma])} \int_{a_{i}}^{a_{i}^{\prime}} d \tilde{a}_{i} .
$$

Dividing the preceding equation by $\int_{a_{i}}^{a_{i}^{\prime}} d \tilde{a}_{i}=a_{i}^{\prime}-a_{i}$ and taking limits produces

$$
\lim _{a_{i}^{\prime} \rightarrow a_{i}} \frac{1}{\bar{\sigma}-\underline{\sigma}} \int_{\underline{\sigma}}^{\bar{\sigma}} \frac{\Delta x(\sigma \mid \cdot)}{a_{i}^{\prime}-a_{i}} d \sigma=\frac{\partial D_{i}(\mathbf{p}, \boldsymbol{\theta})}{\partial a_{i}},
$$

which completes the proof.

PROOF OF PROPOSITION 4: For convenience, we let $\hat{\tau} \equiv \tau-\mu \mathbb{E}[\sigma]$ and recall that the (inverse) substitutability index $\hat{\tau} \equiv \tau-\mu \mathbb{E}[\sigma]$ is positive by assumption. The first-order conditions of the problems $\max _{a_{i}} \pi_{i}(\mathbf{a})-\beta a_{i}^{2}$ are, in matrix form, given by

$$
\left(\begin{array}{cc}
-\omega^{2}+18 \hat{\tau} \beta & \omega^{2} \\
-\omega^{2} & \omega^{2}-18 \hat{\tau} \beta
\end{array}\right)\left(\begin{array}{l}
a_{1} \\
a_{2}
\end{array}\right)=\omega\left(\begin{array}{l}
\gamma+3 \hat{\tau} \\
\gamma-3 \hat{\tau}
\end{array}\right),
$$


where $\gamma \equiv q_{1}-q_{2}-c_{1}+c_{2}$. The matrix on the LHS of (A.3), call it $M$, is invertible if and only if $\operatorname{det} M \neq 0$; that is, if and only if $324 \hat{\tau}^{2} \beta^{2}-36 \hat{\tau} \beta \omega^{2} \neq 0$. This condition holds whenever $\beta>\omega^{2} / 9 \hat{\tau}$. The reduced-form profit function is strictly concave in own advertising if $\beta>\omega^{2} / 18 \hat{\tau}$. ${ }^{11}$ If the invertibility condition holds, the first-order conditions uniquely determine the advertising levels, and hence the solutions of the system of first-order condition in (A.3) are given by

$$
a_{i}^{*}=\frac{\omega}{2}\left(\frac{1}{3 \beta}+\frac{\left(q_{i}-c_{i}\right)-\left(q_{j}-c_{j}\right)}{9 \beta \hat{\tau}-\omega^{2}}\right) .
$$

The advertising intensities $a_{i}^{*}$ are positive as long as $|\gamma|<\left(9 \beta \hat{\tau}-\omega^{2}\right) / 3 \beta$. Substituting $a_{i}^{*}$ into (1) and plugging the $\theta_{i}$ 's into (7) yields the optimal prices

$$
p_{i}^{*}=\hat{\tau}+c_{i}+\frac{3 \beta \hat{\tau}\left(\left(q_{i}-c_{i}\right)-\left(q_{j}-c_{j}\right)\right)}{9 \beta \hat{\tau}-\omega^{2}} .
$$

This completes the proof.

PROOF OF OBSERVATION 1: First, consider advertising intensities. From (9),

$$
a_{i}^{*}-a_{j}^{*}=\frac{\omega\left(\left(q_{i}-c_{i}\right)-\left(q_{j}-c_{j}\right)\right)}{9 \beta \hat{\tau}-\omega^{2}} .
$$

The invertibility condition (Proposition 4) requires that $9 \beta \hat{\tau}-\omega^{2}>0$. Thus, firm $i$ has a higher advertising intensity if and only if it has a higher qualitycost margin.

Next, consider pricing. From (10),

$$
p_{i}^{*}-p_{j}^{*}=\frac{6 \beta \hat{\tau}\left(q_{i}-q_{j}\right)-\left(\omega^{2}-3 \beta \hat{\tau}\right) c_{i}+\left(\omega^{2}-3 \beta \hat{\tau}\right) c_{j}}{9 \beta \hat{\tau}-\omega^{2}} .
$$

Write the numerator of (A.5) as $\left(6 \beta \hat{\tau} q_{i}-\left(\omega^{2}-3 \beta \hat{\tau}\right) c_{i}\right)-\left(6 \beta \hat{\tau} q_{j}-\left(\omega^{2}-3 \beta \hat{\tau}\right) c_{j}\right)$. By the hypothesis, $\left(q_{i}-c_{i}\right)-\left(q_{j}-c_{j}\right)>0$. As $9 \beta \hat{\tau}-\omega^{2}>0$, both the numerator and the denominator of (A.5) are positive. Thus, $p_{i}^{*}-p_{j}^{*}>0$, which completes the proof.

PROOF OF OBSERVATION 2: The equilibrium markups $m_{i}^{*}=p_{i}^{*}-c_{i}$ follow from (10), and they are given by

$$
m_{i}^{*}=\hat{\tau}\left(1+\frac{3 \beta\left(\left(q_{i}-c_{i}\right)-\left(q_{j}-c_{j}\right)\right)}{9 \beta \hat{\tau}-\omega^{2}}\right) .
$$

\footnotetext{
${ }^{11}$ Clearly, the invertibility condition is more stringent than the second-order condition.
} 
By the hypothesis and using the invertibility condition (Proposition 4), we have that $m_{i}^{*}>m_{j}^{*}$. Now, plugging the price $p_{i}(\boldsymbol{\theta})$ from (7) into the demand function as given in (6) yields $D_{i}(\boldsymbol{\theta})=\left(3 \hat{\tau}+\left(\theta_{i}-\theta_{j}\right)-\left(c_{i}-c_{j}\right)\right) / 6 \hat{\tau}$. Next, from (7), the markups as a function of $\boldsymbol{\theta}$ are given by $m_{i}(\boldsymbol{\theta})=\left(3 \hat{\tau}+\left(\theta_{i}-\theta_{j}\right)-\left(c_{i}-c_{j}\right)\right) / 3$. Thus, $m_{i}(\boldsymbol{\theta})=2 \hat{\tau} D_{i}(\boldsymbol{\theta})$, which implies that $D_{i}^{*}>D_{j}^{*}$. Moreover, as $\pi_{i}(\boldsymbol{\theta})=$ $m_{i}(\boldsymbol{\theta}) D_{i}(\boldsymbol{\theta})$, we have that $\pi_{i}^{*}>\pi_{j}^{*}$. Finally, letting $\Pi_{i}(\mathbf{a}) \equiv \pi_{i}(\mathbf{a})-\beta a_{i}^{2}$, straightforward substitution from (9) yields

$$
\Pi_{i}^{*}=\frac{1}{2}\left(k-\frac{\omega^{2}}{18 \beta}\right)\left(1+\frac{3 \beta\left(\left(q_{i}-c_{i}\right)-\left(q_{j}-c_{j}\right)\right)}{9 k \beta-\omega^{2}}\right)^{2} .
$$

Invoking the hypothesis and the invertibility condition again, we immediately have that $\Pi_{i}^{*}>\Pi_{j}^{*}$. This completes the proof.

PROOF OF OBSERVATION 3: Rewrite $p_{i}^{*}$ as given in (9) as

$$
p_{i}^{*}=\hat{\tau}+\frac{6 \beta \hat{\tau} \omega}{\omega}\left(\frac{1}{6 \beta}+\frac{\left(q_{i}-c_{i}\right)-\left(q_{j}-c_{j}\right)}{2\left(9 \beta \hat{\tau}-\omega^{2}\right)}\right)-\hat{\tau}+c_{i} .
$$

Substituting $a_{i}^{*}$ from (10) into the preceding equation yields $p_{i}^{*}=6 \beta \hat{\tau} a_{i}^{*} / \omega+c_{i}$, which implies a positive relationship between advertising intensities and pricing. This completes the proof.

PROOF OF OBSERVATION 4: Differentiating (A.4) with respect to $\mathbb{E}[\sigma]$ and using the invertibility condition (from Proposition 4) immediately yields that $\partial\left(a_{i}^{*}-a_{j}^{*}\right) / \partial \mathbb{E}[\sigma]>0$. Now, differentiating (A.4) with respect to $\hat{\tau}$ yields

$$
\operatorname{sign}\left(\frac{\partial\left(p_{i}^{*}-p_{j}^{*}\right)}{\partial \hat{\tau}}\right)<0 .
$$

Observing that $\partial \hat{\tau} / \partial \mathbb{E}[\sigma]<0$, this inequality and (A.6) together imply that $\partial\left(p_{i}^{*}-p_{j}^{*}\right) / \partial \mathbb{E}[\sigma]>0$, which completes the proof.

Remark: The effects of $\mu$ are qualitatively the same as those of $\mathbb{E}[\sigma]$. The impact on the price gap follows by noting that $\partial \hat{\tau} / \partial \mu<0$.

PROOF OF OBSERVATION 5: Differentiating (A.4) with respect to $\omega$ and using the invertibility condition (from Proposition 4 ) yields that $\partial\left(a_{i}^{*}-a_{j}^{*}\right) / \partial \omega>0$. Next, observing that $\partial\left(p_{i}^{*}-p_{j}^{*}\right) / \partial \omega>0$ establishes the result. 


\section{References}

Amaldoss, W. And S. Jain (2005): "Pricing of Conspicous Goods: A Competitive Analysis," Journal of Marketing Research, XLII, 30-42.

Anderson, S. P. AND S. COATE (2005): “Market Provision of Broadcasting: A Welfare Analysis," The Review of Economic Studies, 72, 947-972.

Anderson, S. P. And A. DE PAlma (2001): "Product Diversity in Asymmetric Oligopoly: Is the Quality of Consumer Goods Too Low?" Journal of Industrial Economics, 49, 113-135.

ATHEY, S. AND A. SCHMUtZLER (2001): "Investment and Market Dominance," RAND Journal of Economics, 32, 1-26.

BAAKE, P. AND A. BoOM (2001): “Vertical Product Differentiation, Network Externalities, and Compatibility Decisions," International Journal of Industrial Organization, 19, 267-284.

BAgWELL, K. (2007): “The Economic Analysis of Advertising," in Handbook of Industrial Organization, ed. by M. Armstrong and R. Porter, North-Holland, vol. III, 1701-1844.

BERNHeIM, B. D. (1994): “A Theory of Conformity," Journal of Political Economy, $102,841-877$.

Clark, A. E. AND A. J. OswAld (1998): “Comparison-Concave Utility and Following Behavior in Social and Economic Settings," Journal of Public Economics, 70, 133-155.

Ellison, G. (2006): “Bounded Rationality in Industrial Organization," in Advances in Economics and Econometrics: Theory and Applications, Ninth World Congress, ed. by R. Blundell, W. K. Newey, and T. Persson, North-Holland, vol. II.

FARrell, J. AND P. Klemperer (2007): “Coordination and Lock-In: Competition with Switching Costs and Network Effects," in Handbook of Industrial Organization, ed. by M. Armstrong and R. Porter, North-Holland, vol. III, 1967-2072. 
Gabszewicz, J. J., D. Laussel, AND N. SonNaC (2004): “Attitudes Toward Advertising and Price Competition in the Press Industry," in Economics of Art and Culture, ed. by V. A. Ginsburgh, Elsevier, 61-74.

(2005): "Does Advertising Lower the Price of Newspapers to Consumers? A Theoretical Appraisal," Economics Letters, 87, 127-134.

Grilo, I., O. ShY, AND J.-F. Thisse (2001): "Price Competition When Consumer Behavior is Characterized by Conformity or Vanity," Journal of Public Economics, 80, 385-408.

Johnson, J. P. AND D. P. MYATt (2006): “On the Simple Economics of Advertising, Marketing, and Product Design," American Economic Review, 96, 756-784.

KAISER, U. AND M. SONG (2009): “Do Media Consumers Really Dislike Advertising? An Empirical Assessment of the Role of Advertising in Print Media Markets," International Journal of Industrial Organization, 27, 292-301.

Karni, E. AND D. SChMeidler (1990): "Fixed Preferences and Changing Tastes," American Economic Review, 80, 262-267.

KATZ, M. L. AND C. Shapiro (1985): "Network Externalities, Competition and Compatibility," American Economic Review, 75, 424-440.

KelLeR, K. L. AND D. R. LeHMANN (2006): “Brand and Branding: Research Findings and Future Priorities," Marketing Science, 25, 740-759.

Laffont, J.-J., P. Rey, And J. Tirole (1998): “Network Competition: I. Overview and Nondiscriminatory Pricing," RAND Journal of Economics, 29, $1-37$.

Peitz, M. And T. M. Valletti (2008): "Content and Advertising in the Media: Pay-TV versus Free-to-Air," International Journal of Industrial Organization, 26, 949-965.

RotemberG, J. J. (2010): “Persuasion and Empathy in Salesperson-Customer Interactions," NBER Working Paper, No. 15975.

Sobel, J. (2005): “Interdependent Preferences and Reciprocity," Journal of Economic Literature, XLIII, 392-436. 
Vandenbosch, M. B. And C. B. Weinberg (1995): "Product and Price Competition in a Two-Dimensional Vertical Differentiation Model," Management Science, 14, 224-249.

VON DER FeHR, N.-H. AND K. STEVIK (1998): "Persuasive Advertising and Product Differentiation," Southern Economic Journal, 65, 113-126. 